\title{
The probability of an event and estimates of posterior probability based upon its occurrence'
}

CHARLES F. GETTYS AND CHARLES W. MANLEY, HUMAN PERFORMANCE CENTER, THE OHIO STATE UNIVERSITY, Columbus, Ohio 43210

The Ss estimated posterior probabilities for a series of problems which had varied a priori probabilities of the datum and likelihood ratios to determine if subjective estimates are influenced by the probability of the datum. No such influence was found in this study or in its subsequent replication, and these results are compared to another study which found an effect.

A class of variables is present in Bayesian inference situations which does not directly enter into the calculation of Bayes' theorem. If Bayes' theorem is to be an acceptable model of human inference, then these variables should not markedly influence human inference. For example, it has been shown that while Bayes' theorem is indifferent to the order in which the conditionally independent data occur, human behavior does reflect this factor (Peterson \& DuCharme, 1967). The present study is concerned with another in this class of variables: the a priori probability of the event or datum on the basis of which inference is made-usually designated P(D). It may be that the confidence that humans have in a hypothesis given the information provided by an event is influenced by the a priori probability of that event. To put it more simply, the question becomes: Is man's judgment about the significance of an event influenced by his perception of its frequency of occurrence?

Bayes' theorem has the property that it is indifferent to the size of $P(D) . P(D)$ is not present in the odds form of Bayes' theorem and serves only as a normalizing constant in the probability form (Edwards, 1966). For example, consider the following two problems shown in Table 1 . In both problems, $A$ and $B$ are two urns in which there are differing numbers of red and white balls.

In the situation used in this experiment, an urn is chosen at random, $P(A)=P(B)=.5$, and $S$ estimates the posterior probabilities or odds that a ball of a particular color came from Urn A or B. The probability of Urn A given that a white ball was chosen, $\mathrm{P}(\mathrm{A} \mid \mathrm{w})$, is .25 for both problems (in odds, $\mathrm{A}: \mathrm{B}=1: 3$ ). If $\mathrm{S}$ 's response is Bayesian, then the difference in $P(D)$ that distinguishes these two problems (the probability of drawing a white ball, $P(w)$, in this case) should not affect S's response. If S's response is affected by $P(D)$, then the difference between the $P(w)$ for Problem 1 and Problem 2 (i.e., .312 and .622, respectively) should be reflected in his estimates.

Method-Group 1. Five values of $P(D)$ ranging from .05 to .55 in equal steps $(.05, .175, .30$, etc.) were combined factorially with five likelihood ratios ranging from $1.5: 1$ to $9.5: 1$ in equal steps $1.5: 1,3.5: 1$, etc.) to produce a total of 25 distinct problems of the type shown in Table 1. By interchanging the rows and the columns of each of the 25 original problems, 100 problems were created. Ten problems were chosen randomly for each page, and bound into a test booklet. The order of pages in the booklet was randomly arranged for each $\mathrm{S}$.

Twenty college-age Ss served in the experiment. Ss were told that the numbers in these problems represented the number of white and red balls in each of two urns, that the a priori probabilities of the urns were equal, and that an arrow indicated the color of the ball drawn. Their task was to estimate the posterior probability (which was explained to them in terms of "confidence") that the ball came from Urn A or B given the color of the ball and the fact that the urns were chosen with equal frequency. They were instructed to make their estimates in three-place decimals within the range of .001 to .999 which summed to 1.000 .

The task was self-paced, but Ss were encouraged to finish in $2 \mathrm{~h}$. Following the completion of the test booklet, each $S$ filled out a questionnaire regarding his familiarity with Bayes' theorem and the features of the problem which influenced his estimates.

Method-Group 2: A Replication. The replication of this experiment was prompted by the discovery that Beach (in press), inspired by a hypothesis of Vlek (1965), had conducted a similar experiment and found that $P(D)$ had an effect on subjective posterior probabilities. ${ }^{2}$ The only procedural difference between this replication and the procedure used in Group 1 was the nature of the restriction placed on the P(D) values. In Method Group 2 P(D) was not fixed as it was in Method Group 1. Instead, four problems were randomly chosen for each of the $25 \mathrm{P}(\mathrm{D})$-odds combinations so that the $\mathrm{P}(\mathrm{D})$ was \pm .025 from the following five values: $.075, .175, .275, .375$, and .475 . The 100 problems thus created had their rows and columns randomly interchanged so as to confound positional response bias. The procedure for the $28 \mathrm{Ss}$ was otherwise the same in the replication.

Results and Discussion. The S's probability estimates were converted to $\log$ odds. The antilogs of the mean log odds (a geometric mean) for S's estimates are shown in Fig. 1 as a function
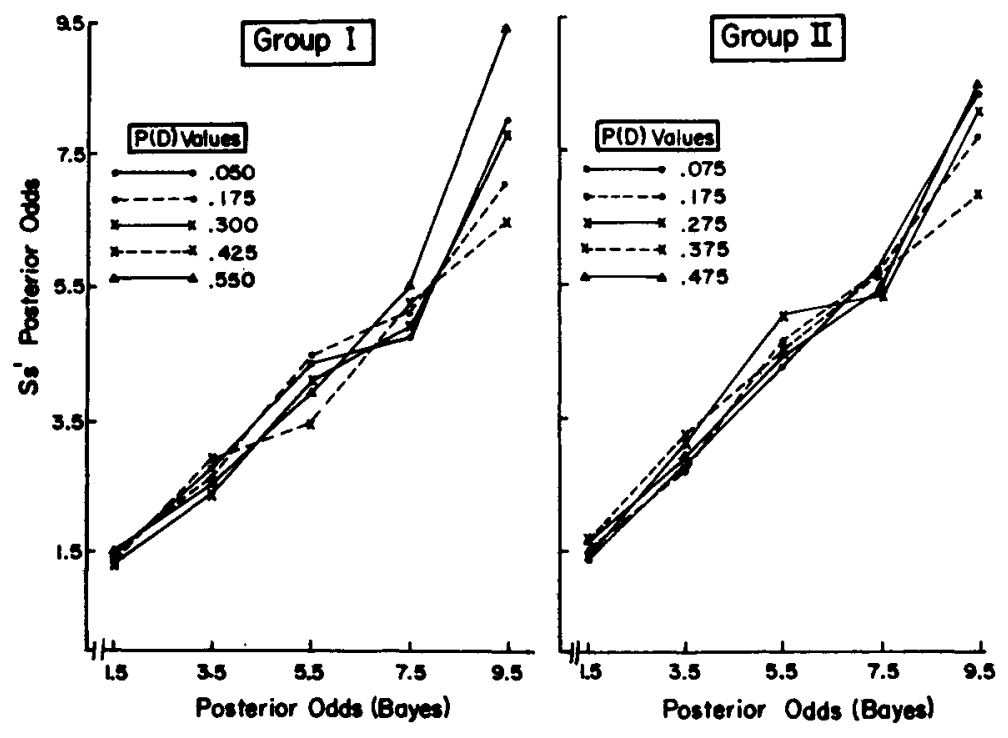

Fig. 1. Mean posterior odds as a function of the posterior odds and P(D). 
Table 1

Sample Problems Which Vary in $\mathbf{P ( D )}$

\begin{tabular}{lrrrr} 
& \multicolumn{2}{c}{ Problem 1 } & & \multicolumn{2}{c}{ Problem 2 } \\
\cline { 2 - 5 } & Urn A & Urn B & Urn A & Urn B \\
\hline Red & 844 & 532 & 689 & 67 \\
White & 156 & 468 & 311 & 933 \\
\hline Total & 1000 & 1000 & 1000 & 1000 \\
\hline
\end{tabular}

of the various Bayesian posterior odds for the various $P(D)$ values Since the prior odds are equal, the Bayes posterior odds are equal to the likelihood ratios of the data in this case.

Analyses of variance applied to these data confirm the fact that the a priori probability of the datum has little, if any, influence on the estimate of posterior probability in either group: Group $1 \mathrm{~F}=$ .56 , df $=4 / 76$; Group $2(F=.18, \mathrm{df}=4 / 108)$. The interaction between $P(D)$ and odds was also insignificant. As might be expected from previous research (Peterson \& Miller, 1965), S's estimates increased significantly with the Bayes posterior odds (Group 1: $\mathrm{F}=98.50, \mathrm{df}=4 / 76 ;$ Group 2: $\mathrm{F}=248.25, \mathrm{df}=$ $4 / 108)$.

A similar analysis using another dependent measure, the accuracy ratio (see Peterson et al, 1965), reinforced the conclusion that S's posterior probability estimates are influenced little, if at all, by the a priori probability of the datum.

As mentioned previously, Beach (in press) found convincing evidence suggesting that the size of $\mathrm{P}(\mathrm{D})$ does affect human posterior probability estimates. His results show that human departures from the optimal Bayes solution were higher when the $P(D)$ associated with the likelihood ratio was small than when it was large. However, the close agreement obtained between the first study and its replication suggests that the present results though in conflict with those of Beach are reliable.

A possible explanation for the discrepancy is based upon the fact that problems contained a different number of data items in the Beach study (three items per problem) and in the present one (one item per problem). This difference means that while Beach's Ss aggregated information from one trial to the next and thus revised their subjective probabilities continuously, Ss in the present study were not required to aggregate at all, and merely translated likelihood ratios into posterior probabilities. The present task is, of course, much simpler. On this basis it might be argued that the Beach results are not due to the fact that $S$ regards likelihood ratios which have small $P(D)$ values as relatively uninformative, as Beach hypothesized, but rather that they stem from a human deficiency in aggregating frequentistic information. In other words, small P(D) values may be considered as relatively uninformative, but only when it is necessary to combine them with, or compare them to, more frequent events. In the present study such comparison was not involved since there was only one datum for each problem.

\section{REFERENCES}

BEACH, L. R. Probability magnitudes and conservative revisions of subjective probabilities. J. exp. Psychol., 1968, in press.

EDWARDS, W. Introduction to special issue on revision of opinions by men and man-machine systems. IEEE Trans. hum. Factors Electron., 1966, HFE-7, 1-6.

PETERSON, C., \& DUCHARME, W. A primacy effect in subjective probability revision. J. exp. Psychol., 1967, 73, 61-65.

PETERSON, C., \& MILLER, A. Sensitivity of subjective probability revision. J. exp. Psychol., 1965, 70, 117-121.

PETERSON, C., SCHNEIDER, R., \& MILLER, A. Sample size and the revision of subjective probabilities $J$. exp. Psychol., 1965, 69, 522-527.

VLEK, C. The use of probabilistic information in decision making. Psychol. Inst Rep., University of Leiden, Netherlands, No. 009-65, 1965.

\section{NOTES}

1. The research reported in this paper was carried out at the Human Performance Center and was supported by the Aeromedical Research Laboratories, Aerospace Medical Division, Air Force Systems Command, Wright-Patterson Air Force Base, Ohio under Contract No. AF 33(615)2248 with the Ohio State University Research Foundation. Further reproduction is authorized to satisfy the needs of the United States Government.

2. Both Beach and Vlek hypothesized that the magnitudes of the numbers in the likelihood ratio influenced S's estimate. However, in their situation and in the present experiment the magnitude of the numbers in the likelihood ratio is simply $2 \mathrm{P}(\mathrm{D})$. Consequently the explanations are completely confounded and equivalent in this situation, and the only prediction that one can make from Beach's results is that either variable may have an effect, as the magnitude of the likelihood ratio and P(D) are only separate entities when there are more than two hypotheses, or when the prior odds are unequal. 